\title{
Sub-picosecond Hot Hole Transfer in a Graphene Quantum Dot Composite with High Efficiency.
}

\author{
Krishna Mishra, Debopam Acharjee, Ayendrila Das, Subhadip Ghosh* \\ School of Chemical Sciences, National Institute of Science Education and Research, Homi Bhabha \\ National Institute (HBNI), Khurda 752050, Odisha, India.
}

*E-mail: sghosh@niser.ac.in

Contents

Experimental Section

Figure S1

Figure S2

Figure S3

Figure S4

Figure S5

Figure S6-S7

Figure S8

Table S1

Table S2

References
Page Number

S2-S4

S5

S6

S7

S8

S9

$\mathrm{S} 10$

S11

S12

S13

S14 


\section{Experimental Section}

\section{Chemicals}

Graphene quantum dots (GQDs; product number 900712, CAS number 7440-44-0), Nmethylaniline (NMA, purity $\sim 99.5 \%$ ) and toluene were purchased from Sigma-Aldrich. GQDs were transferred from water to toluene using partitioning method. In brief, aqueous solution of GQDs was mixed with desired volume of toluene, and the solvent mixture was vortexed before settling down overnight under nitrogen environment. Toluene portion, clearly separated from water, was slowly taken out using a pipette. This toluene solution of GQDs was further diluted with toluene until optical density reached to $<0.3$ for optical studies.

\section{Instruments Used}

Steady state absorption and emission spectra were recorded in Jasco V-730 spectrophotometer and cary eclipse fluorescence spectrophotometer (Agilent Instruments), respectively.

Long lifetime components were measured using a TCSPC setup from Edinburgh Instruments (Model OB-920). 375 $\mathrm{nm}$ and $\sim 45 \mathrm{~nm}$ diode lasers were used for the excitation. Emissions were collected at magic angle $\left(54.7^{\circ}\right)$. IRF $(\sim 0.7 \mathrm{~ns})$ was measured by a scatter ludox solution. Lifetime profiles were fitted using F980 software, provided with the instrument.

Femtosecond fluorescence transients were recorded using a commercially available set-up FluoMax from IB Photonics Ltd. Ti-sapphire laser (Mai Tai HP, Spectra Physics) was used as the excitation source. Fundamental laser beam (of $\sim 750 \mathrm{~nm}$ and $\sim 860 \mathrm{~nm}$ ) with a repetition rate of 80 $\mathrm{MHz}$ was focused onto a BBO crystal, which generates frequency doubled excitation beam (of $\sim 375 \mathrm{~nm}$ and $\sim 430 \mathrm{~nm}$ ) with same repetition rate. Frequency doubled excitation beam was separated from the gate beam using a dichroic mirror. Fluorescence was collected at the magic angle and subsequently up-converted with the gate pulse on a nonlinear BBO crystal. Optical delay line was used to delay the gate pulse with respect to the fluorescence. Dispersed up-converted light 
is guided to a monochromator and finally to the detector. Excitation profile was obtained from the Raman scattering of ethanol. Femtosecond fluorescence transients were fitted with a reconvolution function where a Gaussian shaped lamp profile ( $\sim 300 \mathrm{fs})$ as obtained from Raman scattering experiment, was used. For our experiment we excited the sample with $\sim 375 \mathrm{~nm}$ and $\sim 430 \mathrm{~nm}$ frequency doubled beam.

Powder XRD measurement was performed in a Bruker Davinci D8 advanced diffractometer $(\mathrm{Cu}-$ K $\alpha$ radiation, $\lambda=1.5418 \AA$ ).

FT-IR spectrum was obtained through a Perkin-Elmer RXI FT-IR spectrophotometer.

High resolution TEM (HRTEM) measurements were performed in a JEOL (JEM-2100, $200 \mathrm{kV}$ ) electron microscope.

AFM image (and height profile) was recorded in JPK BioAFM (Bruker). Aqueous solution of GQDs was drop-casted onto a glass side and vacuum dried before measurement. Measurement was performed in noncontact tapping mode at room temperature.

All experiments were performed at room temperature $\left(\sim 20{ }^{\circ} \mathrm{C}\right)$, unless otherwise mentioned.

\section{Chemical Driving Force Calculation}

Chemical driving force $\Delta G$ of hole transfer reaction from photoexcited GQD to ground state NMA ( $E_{o x}^{N M A} \sim 5.49 \mathrm{eV}$ vs. vacuum) is calculated from the following equation. ${ }^{1}$

$$
\Delta G_{P E T}=E_{o x}^{N M A}-\left(E_{r e d}^{G Q D}+E_{00}^{G Q D}\right)
$$

Where, $E_{o x}^{N M A}$ and $E_{\text {red }}^{G Q D}$ are the oxidation potential (HOMO energy) of NMA and the reduction potential (conduction band energy) of GQD. $\mathrm{E}_{00}$ is the band gap energy of GQD. Value of $E_{\text {red }}^{G Q D}$ 
$(3.7 \mathrm{eV} \mathrm{v} / \mathrm{s} \text { vacuum })^{2}$ is obtained from literature, while $\mathrm{E}_{00}(\sim 2.55 \mathrm{eV})$ is estimated from the crosssection of absorption and emission spectra. The value of $\Delta \mathrm{G}$ was found to be $-0.76 \mathrm{eV}$; negative value implying the exergonic nature of charge transfer. 


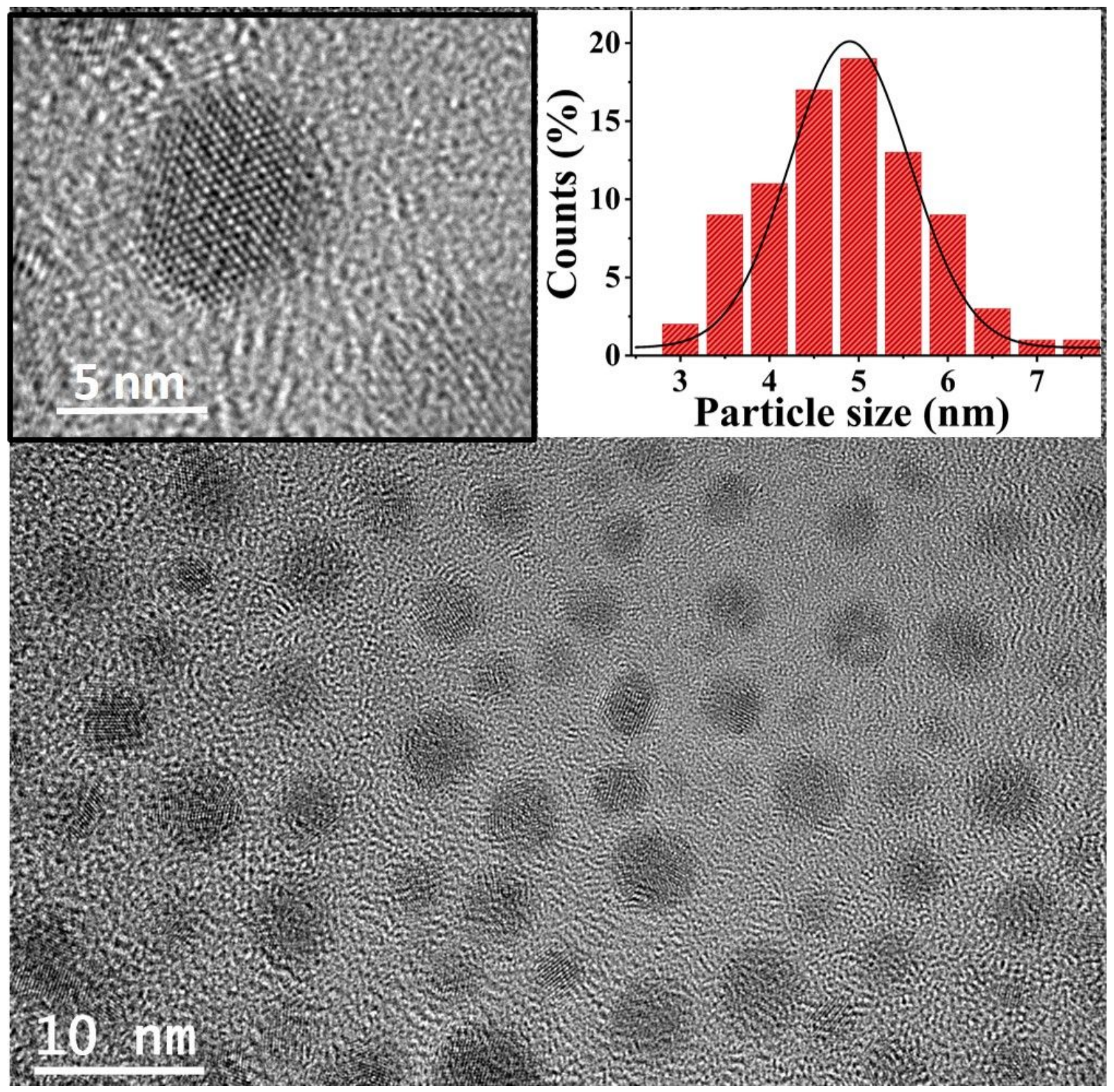

Figure S1. Particle size distribution $(\sim 5.1 \pm 2 \mathrm{~nm})$ of GQDs obtained from HRTEM study. 

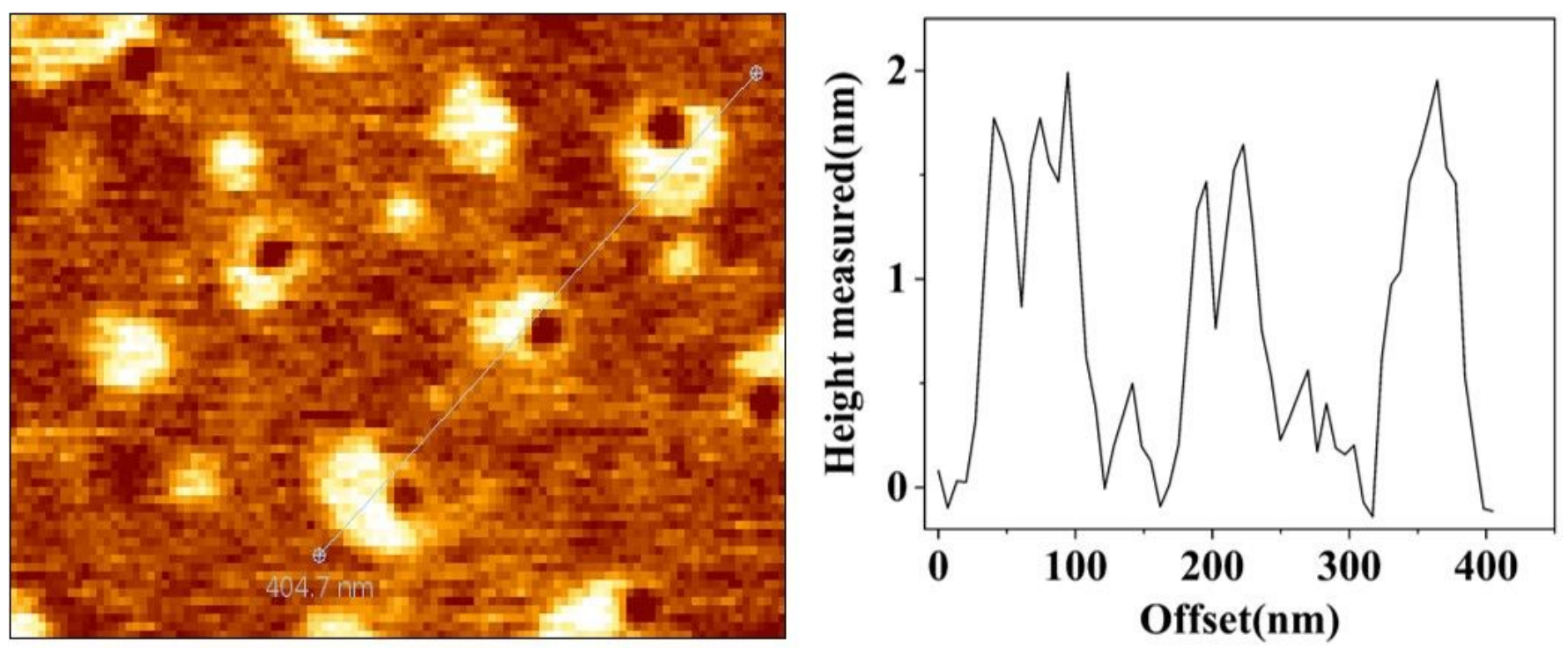

Figure S2. Topological height of the GQDs is measured to be $\sim 1.8 \mathrm{~nm}$ from AFM study, indicating the presence of $\sim 4-5$ graphene layers. 


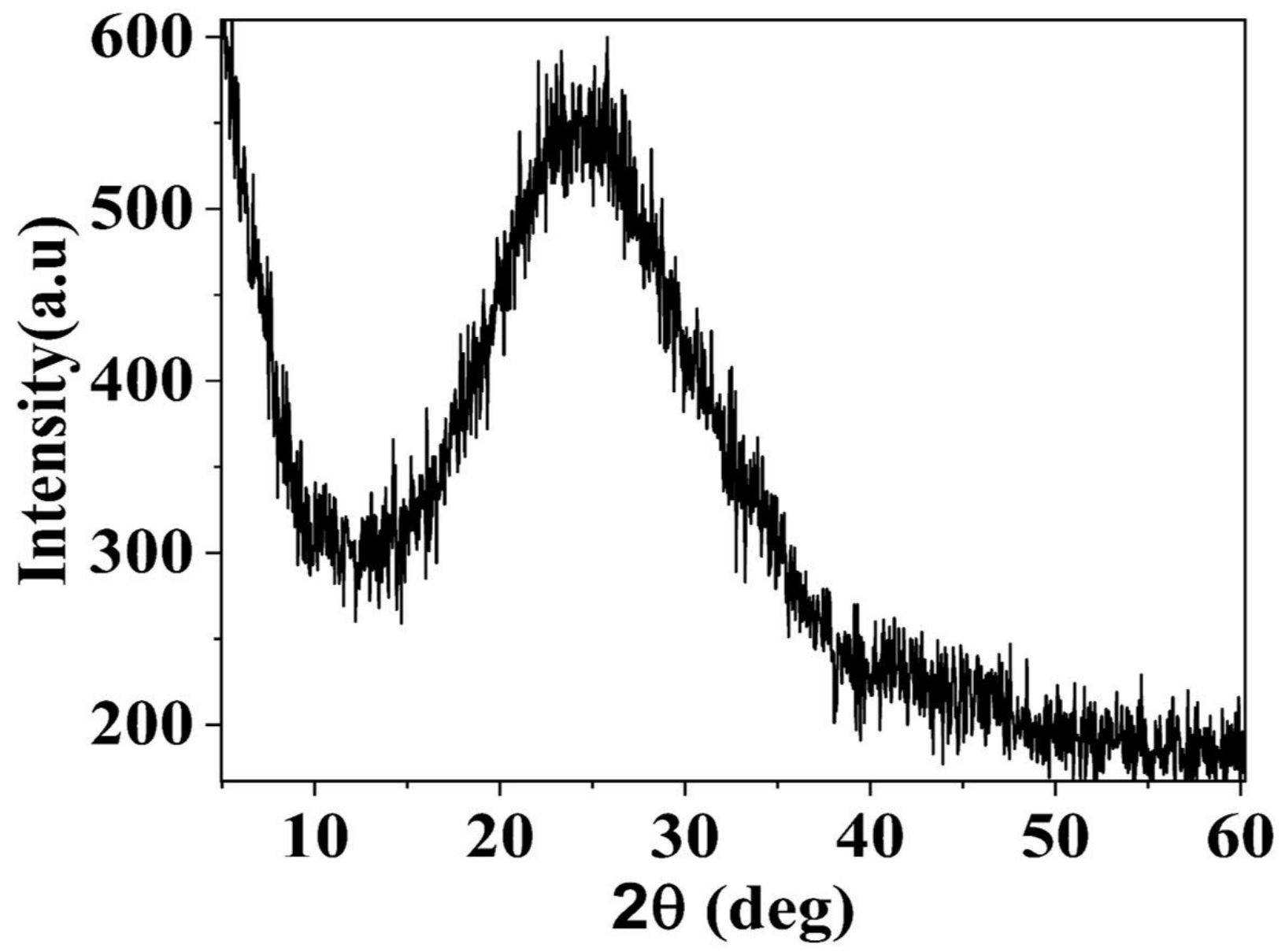

Figure S3. Powder XRD spectrum of GQDs. A diffraction peak was observed at $\sim 24^{0}$ corresponds to the distance between two basal planes of graphene $\sim 0.38 \mathrm{~nm}$. 


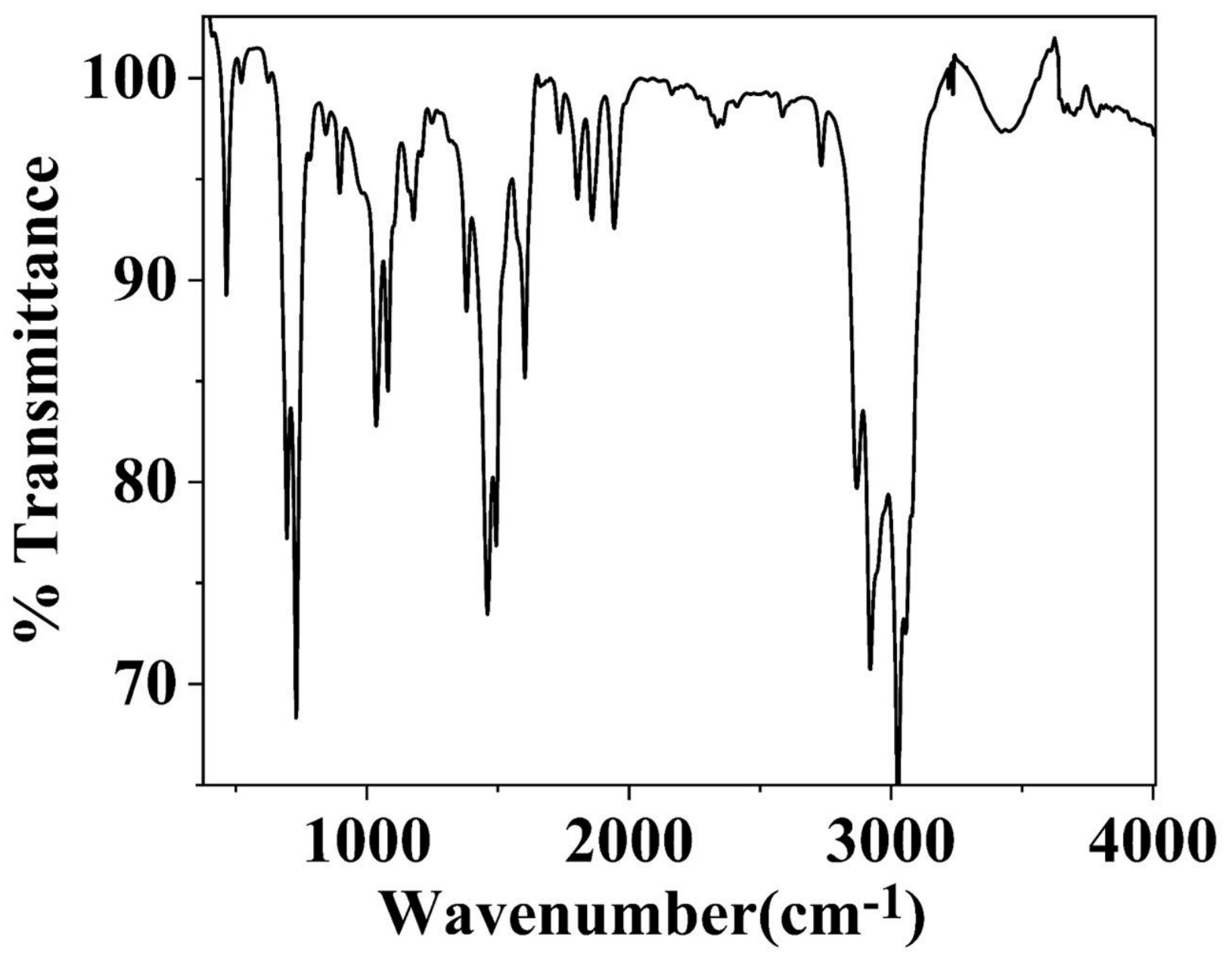

Figure S4. FTIR spectrum of GQDs showing the presence of different organic functional groups on the surface. 


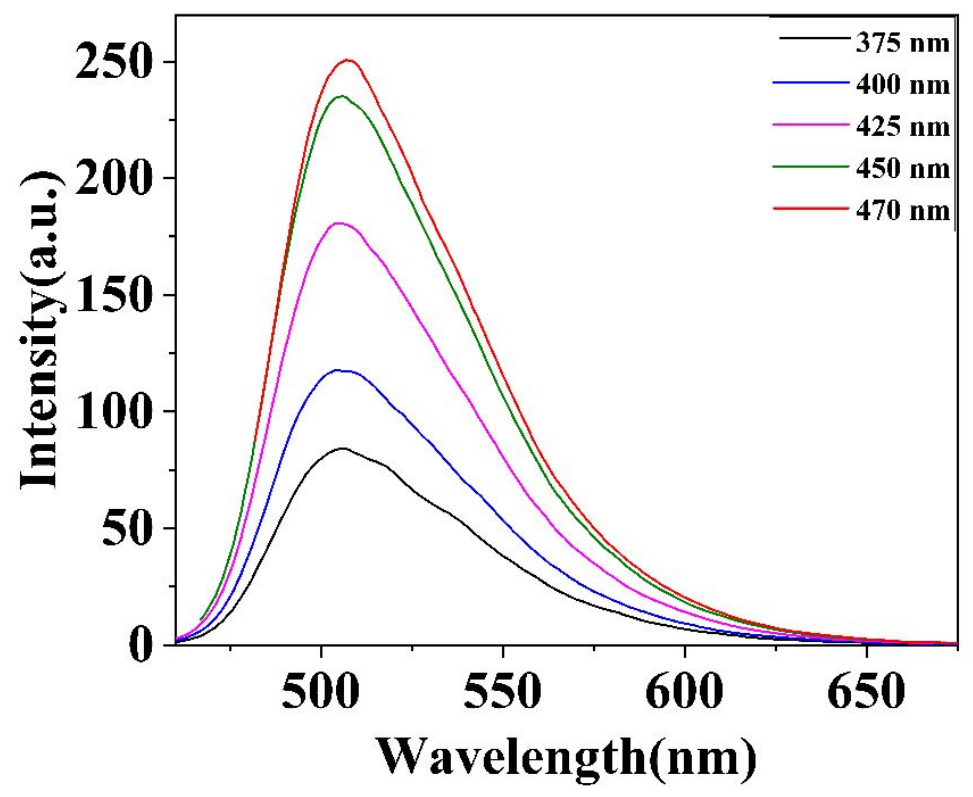

Figure S5. Emission spectra of GQDs in toluene at different excitation wavelengths. 

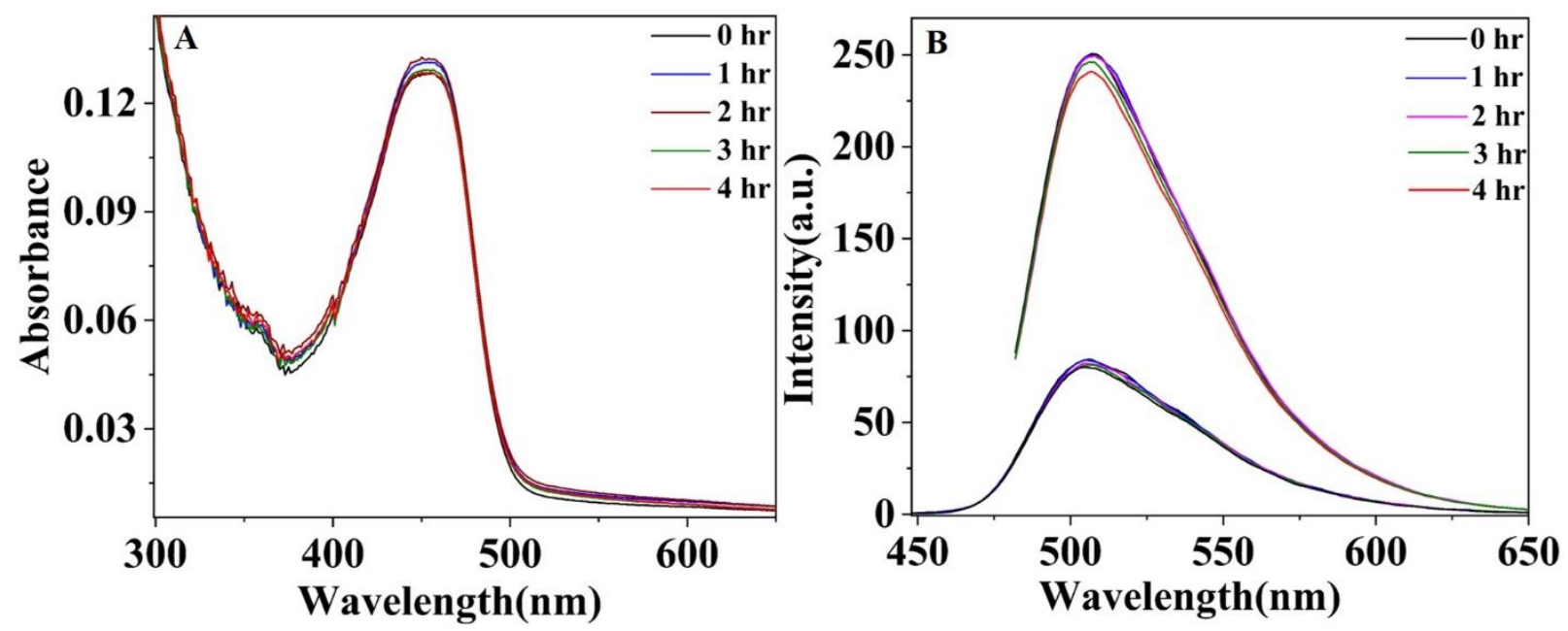

Figure S6. Absorption (left) and emission (right) spectra of GQDs dispersed in toluene at different stages of purification: crude sample without sonication and membrane dialysis $(0 \mathrm{hr})$, after $1 \mathrm{hr} / 2$ $\mathrm{hr} / 3 \mathrm{hr} / 4 \mathrm{hr}$ sonication followed by membrane dialysis. Emission spectra were collected at two different excitations (375 $\mathrm{nm}$ and $470 \mathrm{~nm}$ ).

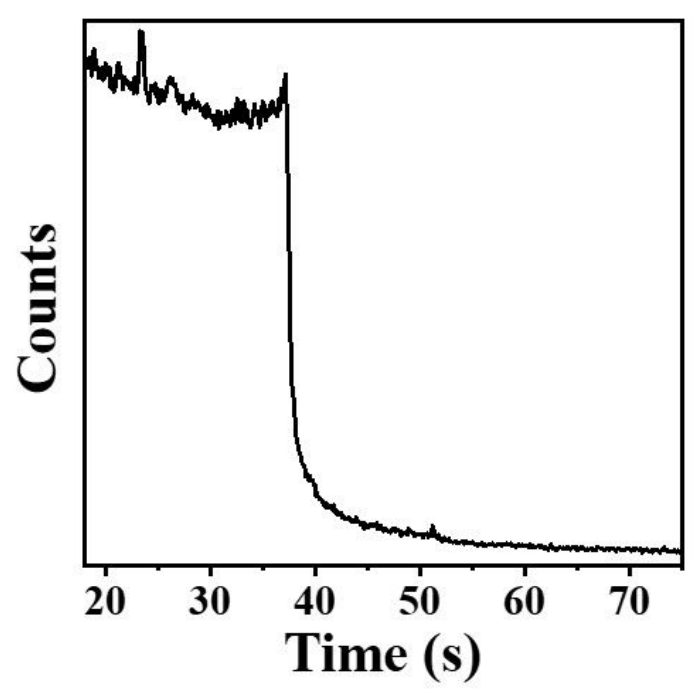

Figure S7. Single step bleaching of single GQD particle, observed in confocal microscope. 

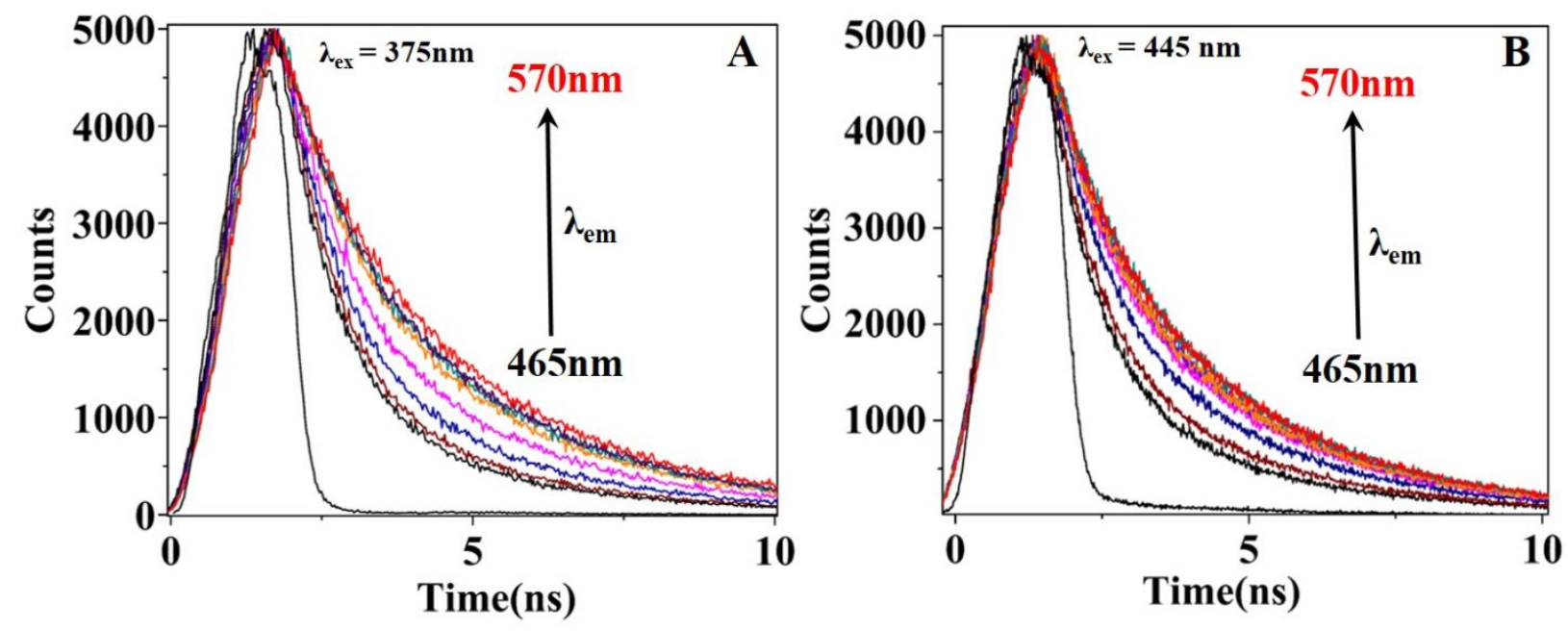

Figure S8. Lifetime profiles of GQDs in toluene recorded in TCSPC at different emission wavelengths (across the emission spectrum) at $375 \mathrm{~nm}(\mathrm{~A})$ and $445 \mathrm{~nm}$ (B) excitations. 
Table S1. Relative PLQY of GQDs (i.e., PLQY of purified/ PLQY of as received) after purification through sonication for different times followed by membrane dialysis.

\begin{tabular}{|l|l|}
\hline Sonication time & Relative PLQY \\
\hline No sonication & 1 \\
\hline $1 \mathrm{hr}$ & $\sim 0.97$ \\
\hline $2 \mathrm{hr}$ & $\sim 1$ \\
\hline $3 \mathrm{hr}$ & $\sim 0.94$ \\
\hline $4 \mathrm{hr}$ & $\sim 0.97$ \\
\hline
\end{tabular}


Table S2. Fluorescence lifetime components of GQDs in tolene at different excitation and emission wavelengths.

\begin{tabular}{|c|c|c|c|c|c|}
\hline$\lambda_{\mathrm{ex}}(\mathrm{nm})$ & $\lambda_{\mathrm{em}}(\mathrm{nm})$ & $\mathbf{a}_{1}$ & $\mathbf{a}_{2}$ & $\tau_{1}(n s)$ & $\tau_{2}(\mathrm{~ns})$ \\
\hline \multirow{11}{*}{375} & 465 & 0.85 & 0.15 & 0.86 & 2.9 \\
\hline & 475 & 0.82 & 0.18 & 0.87 & 2.87 \\
\hline & 485 & 0.75 & 0.25 & 0.91 & 2.96 \\
\hline & 495 & 0.63 & 0.37 & 0.92 & 2.99 \\
\hline & 505 & 0.5 & 0.5 & 1.025 & 3.05 \\
\hline & 515 & 0.43 & 0.57 & 0.97 & 3.03 \\
\hline & 525 & 0.43 & 0.57 & 0.93 & 3.02 \\
\hline & 535 & 0.4 & 0.6 & 0.98 & 3.04 \\
\hline & 545 & 0.38 & 0.62 & 1.08 & 3.10 \\
\hline & 555 & 0.32 & 0.68 & 1.03 & 3.07 \\
\hline & 570 & 0.36 & 0.64 & 1.04 & 3.13 \\
\hline \multirow{11}{*}{445} & 465 & 0.84 & 0.16 & 0.78 & 2.75 \\
\hline & 475 & 0.84 & 0.16 & 0.79 & 2.77 \\
\hline & 485 & 0.74 & 0.26 & 0.72 & 2.53 \\
\hline & 495 & 0.61 & 0.39 & 0.73 & 2.57 \\
\hline & 505 & 0.5 & 0.5 & 0.73 & 2.58 \\
\hline & 515 & 0.44 & 0.56 & 0.72 & 2.56 \\
\hline & 525 & 0.44 & 0.56 & 0.72 & 2.56 \\
\hline & 535 & 0.44 & 0.56 & 0.74 & 2.59 \\
\hline & 545 & 0.38 & 0.62 & 0.7 & 2.56 \\
\hline & 555 & 0.38 & 0.62 & 0.73 & 2.59 \\
\hline & 570 & 0.4 & 0.6 & 0.74 & 2.63 \\
\hline
\end{tabular}




\section{References}

1. Ghosh, T.; Chatterjee, S.; Prasad, E. Photoinduced Electron Transfer from Various Aniline Derivatives to Graphene Quantum Dots. J. Phys. Chem. A 2015, 119, 11783-11790.

2. Yan, X.; Li, B.; Cui, X.; Wei, Q.; Tajima, K.; Li, L.-S. Independent Tuning of the Band Gap and Redox Potential of Graphene Quantum Dots. J. Phys. Chem. Lett. 2011, 2, 1119- 1124. 